\title{
Bolton discrepancy in an Iranian population and its relation with maxillary lateral incisors' size
}

\author{
Shabnam Ajami ${ }^{1}$, Hamidreza Fattahi ${ }^{2}$, Mahsa Zare ${ }^{3}$, Peyman Jenabi ${ }^{4}$
}

${ }^{1}$ DMD, MScD., Assistant Professor of Orthodontics, Othodontic Research Centre, Department of Orthodontics, School of Dentistry, Shiraz University of Medical Sciences, Shiraz, Iran

${ }^{2}$ DMD, MScD., Orhodontic Research Centre, Orthodontist, Private Practice, Shiraz, Iran

${ }^{3}$ Postgraduate Student, Department of Pediatric Dentistry, School of Dentistry, Shiraz University of Medical Sciences, Shiraz, Iran

${ }^{4}$ Postgraduate Student of Endodontics, Department of Endodontics, School of Dentistry, Shiraz University of Medical Sciences, Shiraz, Iran

\section{Type of article: Original}

\begin{abstract}
Background: Bolton's two main ratios describing the proportional size of upper and lower teeth, could contribute to estimating the excess or deficiency of tooth size necessary to obtain an ideal occlusion. However, the mean Bolton values are not the same among different societies. Determining the prevalence of tooth size deviations from population-specific Bolton indices might help local orthodontists to have a more concise treatment plan.

Objective: The study aimed to define the prevalence of clinically significant tooth size discrepancies (TSD) in an Iranian population and to evaluate the influence of lateral incisors' size on this discrepancy.

Methods: This cross-sectional study was conducted on study casts of orthodontic patients attending Imam Reza Dental Clinic from September 2008 to December 2016. The sample comprised of 150 randomly selected pretreatment study casts (64 males and 86 females from 17 to 28). The mesiodistal diameter of all permanent teeth from the first molar on the right to the first molar on the left was measured using 2 similar digital calipers, and Bolton analysis was calculated. Subjective visual estimation of Bolton discrepancy was also performed. SPSS v18.0, Wilcoxon signed ranks test, Pearson correlation and Receiver Operating Characteristic (ROC) curve analysis were used for statistical analysis. A $\mathrm{p}<0.05$ was considered statistically significant.

Results: In the sample group, 34.7\% had anterior Bolton index (ABI) and 20.7\% had total Bolton index (TBI) greater than 2 Standard Deviations (2SDs) of Bolton's means, and about half of them required correction of the ABI considering the actual size of discrepancies $(\mathrm{mm})$. The sensitivity of estimating clinically significant tooth size discrepancy more than 2SDs of Bolton's ABI and the visual judgment was $96.0 \%$ and a cut-off point of $0.12 \mathrm{~mm}$ was obtained.

Conclusion: Bolton's analysis should be routinely performed in all orthodontic patients, and visual estimation of TSD would be suggested as a screening method in the first visit prior to measurements and set-ups.

Keywords: Clinical significance, Tooth size discrepancy, Lateral incisor, Iran
\end{abstract}

\section{Introduction}

Orthodontic treatment includes stages with unique features and challenges. Finishing stage is considered as the one with a lot of details necessary to achieve a perfect occlusion (1). Many orthodontic problems during the finishing stages of treatment can be prevented by determining tooth-size imbalances during initial diagnosis (2). To have a final perfect overjet and overbite and intermaxillary occlusion, early detection of intermaxillary tooth size discrepancy is necessary (3). Moreover, analyzing the relationship of the anterior and total ratio values would indicate in which segment of the arch, the discrepancy might lie. In addition, calculating size ratios in extraction

\section{Corresponding author:}

Dr. Peyman Jenabi. Department of Endodontics, School of Dentistry, Shiraz University of Medical Sciences, Shiraz, Iran. Tel: +98.7136263192, Email: Jenabi@sums.ac.ir

Received: March 25, 2017, Accepted: June 02, 2017, Published: March 2018

iThenticate screening: May 31, 2017, English editing: January 12, 2018, Quality control: January 15, 2018

(C) 2018 The Authors. This is an open access article under the terms of the Creative Commons Attribution-NonCommercialNoDerivs License, which permits use and distribution in any medium, provided the original work is properly cited, the use is non-commercial and no modifications or adaptations are made. 
cases would be an easy practical diagnostic key to choose the most proper teeth for extraction purposes (4). A tooth size discrepancy (TSD) is traditionally expressed as "more tooth structure existing in an arch compared to the other one" (5). It can also be defined as a mismatch between the sizes of individual teeth (6). The well-known study of TSD was performed by Bolton (1), who evaluated 55 cases with excellent occlusions, and developed two ratios for estimating TSD. He defined the anterior Bolton index (ABI) of $77.2 \pm 1.65 \%$ and total Bolton index (TBI) of $91.3 \pm 1.91 \%$. Following this, many studies suggested that different racial and ethnic groups should be treated according to their own characteristics, not exactly to Bolton's ratios $(3,7-10)$. The ABI and TBI among Tabrizian school children were $78.10 \pm 0.28$ and $92.24 \pm 0.21$, respectively. There was no statistically significant difference with Bolton's standard indices (11). Another study in an Iranian-Azari population stated the similar tooth size ratios to the original ratios of an American population, without any gender specificity (12). A study in Shiraz, Iran has also determined Bolton's ratios for the local population in 2006, and declared no statistical difference with Bolton's original indices; ABI of $79.01 \%$ and TBI of $91.68 \%$ were reported (13). Another issue evaluated, was the size of lateral incisors as an index for anterior TSD. Proffit believes that a quick look at the size of maxillary and mandibular lateral incisors could be used to identify the mismatch of upper and lower anterior teeth size for screening purposes (6). A similar study on this subject has also shown that in anterior abnormalities of maxillary teeth, the small size of the lateral incisors could be seen as the cause (14). To the best of knowledge, few studies assessed the accuracy of the content, also, due to the widespread clinical application of this issue; we decided to give it a scientific and rational proof in our study. Proffit's assertion was tested in a way that an expert orthodontist compares the size of maxillary lateral incisor with the mandibular one through visual judgment. Our hypothesis was that a quick look at the size of lateral incisors could be a predictable index to estimate the anterior Bolton discrepancies at the screening stage.

\section{Material and Methods}

\subsection{Setting and selection criteria}

This cross-sectional study was conducted on initial study casts of the orthodontic patients attending Imam Reza Dental Clinic from September 2008 to December 2016. Pretreatment study casts were evaluated for the following criteria:

1) Highly qualified study casts, with no bubbles or fractures

2) All permanent teeth from the left side first molar to the first molar on the right side must have been fully erupted and present in the cast.

3) No extraction and no prior stripping or grinding of teeth should have been reported in the documents.

4) Minor occlusal or buccal/lingual restorations were acceptable.

Exclusion criteria for this study were as follows:

1) Broken study casts

2) Presence of any interdental restorations, build ups, full crowns, onlay or any other prosthetic restorations.

3) Congenital impacted teeth, and teeth with proximal carious lesions.

Among casts representing the mentioned criteria, 150 sets were finally selected using a random-number table, including 64 males and 86 females with the age range of 17 to 28 .

\subsection{Data collection and procedures}

The measurements were carried out using two similar digital calipers with $0.01 \mathrm{~mm}$ accuracy (Mitutoyo Co, Tokyo, Japan). The width of each tooth was measured from mesial contact point to contact point at the greatest distance on the distal contour with the caliper oriented perpendicular to the longitudinal axis of each tooth. This process was repeated for each tooth from the first molar on one side to the other side and for both upper and lower arches of each set of casts. Each measurement was done by two investigators (graduated dentists; M.Z and P.J) and the average of these two measurements was recorded. Only 8-10 casts were evaluated each day in order to avoid fatigue effects. All data from the first molar teeth on one side to the other side were tabulated. ABI and TBI were determined according to Bolton's study (1). The difference of two standard deviations from the mean ( $\pm 2 \mathrm{SDs}$ ) were considered as clinically significant TSD (15). The prevalence of clinically significant anterior and overall TSD was obtained. To obtain intra examiner error, 30 casts were randomly selected from formerly analyzed casts within a week. To ascertain the accuracy of measurements, the inter examiner error was also assessed. In the last step, to evaluate the visual estimation for anterior TSD with a quick look, as described by Proffit (6), the measured casts were reviewed by a single-blind expert orthodontist (Sh. A) in order to check the existence of anterior discrepancies. If anterior discrepancy was assumed by the quick look method, score 1 was given and in the absence of it, score 0 was given. These values were analyzed statistically to determine the specificity and sensitivity of the test and a clinical cut off point. 


\subsection{Statistical analysis}

Data were imported to the SPSS version 18.0 (SPSS Inc., Chicago, IL., USA) to determine the amount of TSDs more than 2SDs of Bolton's means. A $p<0.05$ was considered statistically significant. The prevalence of TSD more than $1.5 \mathrm{~mm}, 2 \mathrm{~mm}$ and $3 \mathrm{~mm}$ was also calculated. To measure inter-examiner reliability, we used the Wilcoxon Signed Ranks Test and Pearson Correlation. To find a cutoff point for the lateral size based on the visual detection, and also to evaluate the sensitivity and specificity of the quick look test, Receiver Operating Characteristic (ROC) curve analysis was used.

\subsection{Ethics}

Both examiners were blind during cast selection. The identity of the patients was confidential and there was no access to the patient's photo or history. Only the initial study casts were used for the study. Ethics approval of the research (No. 2017-333) was granted by the "Local Research Ethics Committee".

\section{Results}

\subsection{Demographic data of the population}

The presented sample included patients from 17 to 28 years old with the average age of 21.3. This population consisted of $43 \%$ males and $57 \%$ females.

\subsection{Size and distribution of TSD}

The distribution of anterior tooth ratios in this study is categorized by Bolton's original means and standard deviations (SD) (Figure 1). In total, $34.7 \%$ of the samples had anterior tooth-width ratios greater than $\pm 2 \mathrm{SD}$ from Bolton's mean ( $32 \%$ greater than $+2 \mathrm{SD}$ and $2.7 \%$ less than $-2 \mathrm{SD})$. This shift to the right, compared with Bolton's results, demonstrates relatively more mandibular tooth-width in comparison to maxilla. For the TBIs, $20.7 \%$ was the reported percentage for ratios more than $\pm 2 \mathrm{SDs}$ of Bolton mean ( $20 \%$ greater than $+2 \mathrm{SD}$ and $0.7 \%$ less than $2 \mathrm{SD})$. (Figure 2). On the other hand, taking $1.5 \mathrm{~mm}$ as the limit of acceptable discrepancy (6), it is revealed that $45.4 \%$ $(40.7 \%+4.7 \%)$ of patients would require correction. Table 1 demonstrates the prevalence of TSD beyond 2 and 3 $\mathrm{mm}$.

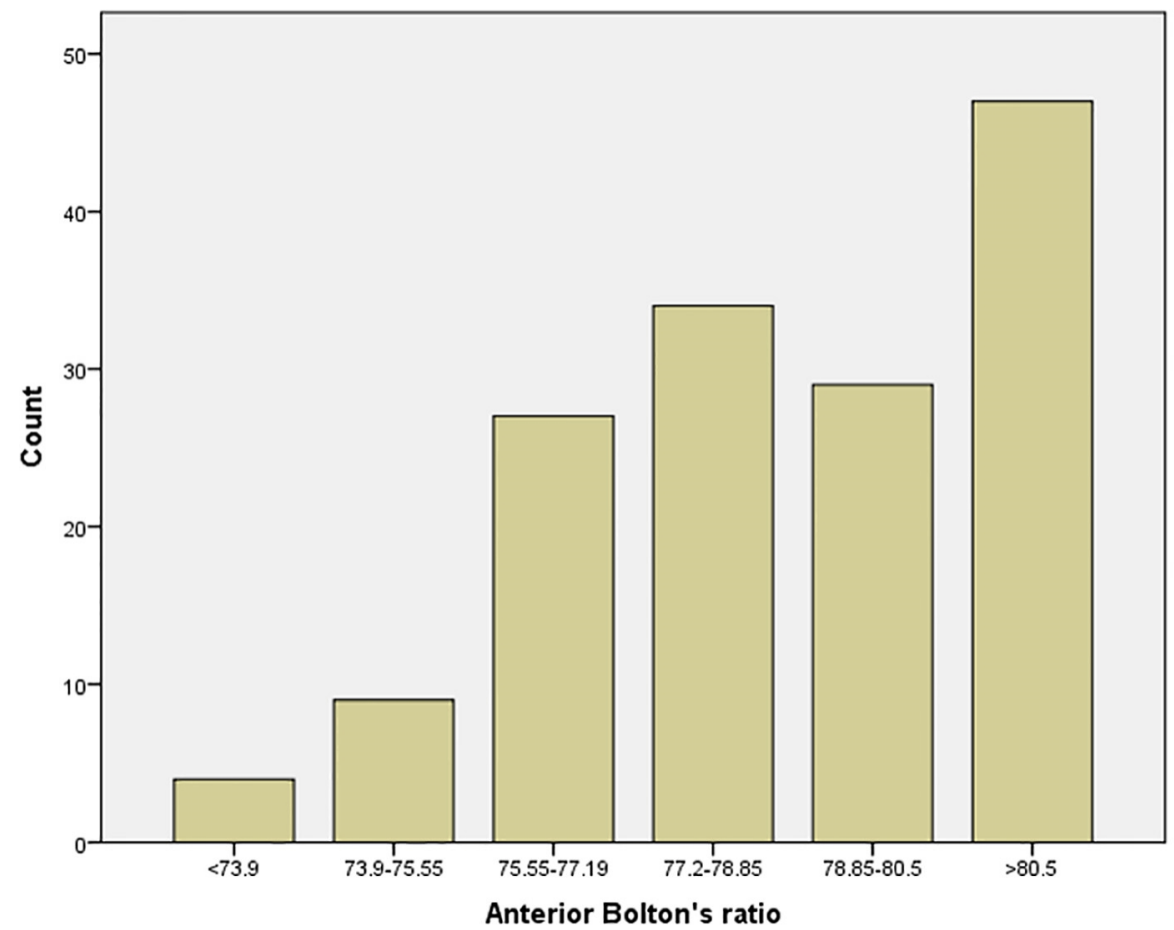

Figure 1. The distribution of anterior tooth ratios in this study categorized by Bolton's original mean and SD

Table 1. The prevalence of TSD beyond $1.5,2$ and $3 \mathrm{~mm}$

\begin{tabular}{|l|l|l|l|l|l|l|}
\hline Discrepancy $(\mathrm{mm})$ & $<-1.5$ & $<-2$ & $<-3$ & $>+1.5$ & $>+2$ & $>+3$ \\
\hline Prevalence $(\%)$ & 40.7 & 30 & 13.3 & 4.7 & 2.7 & 1.3 \\
\hline
\end{tabular}




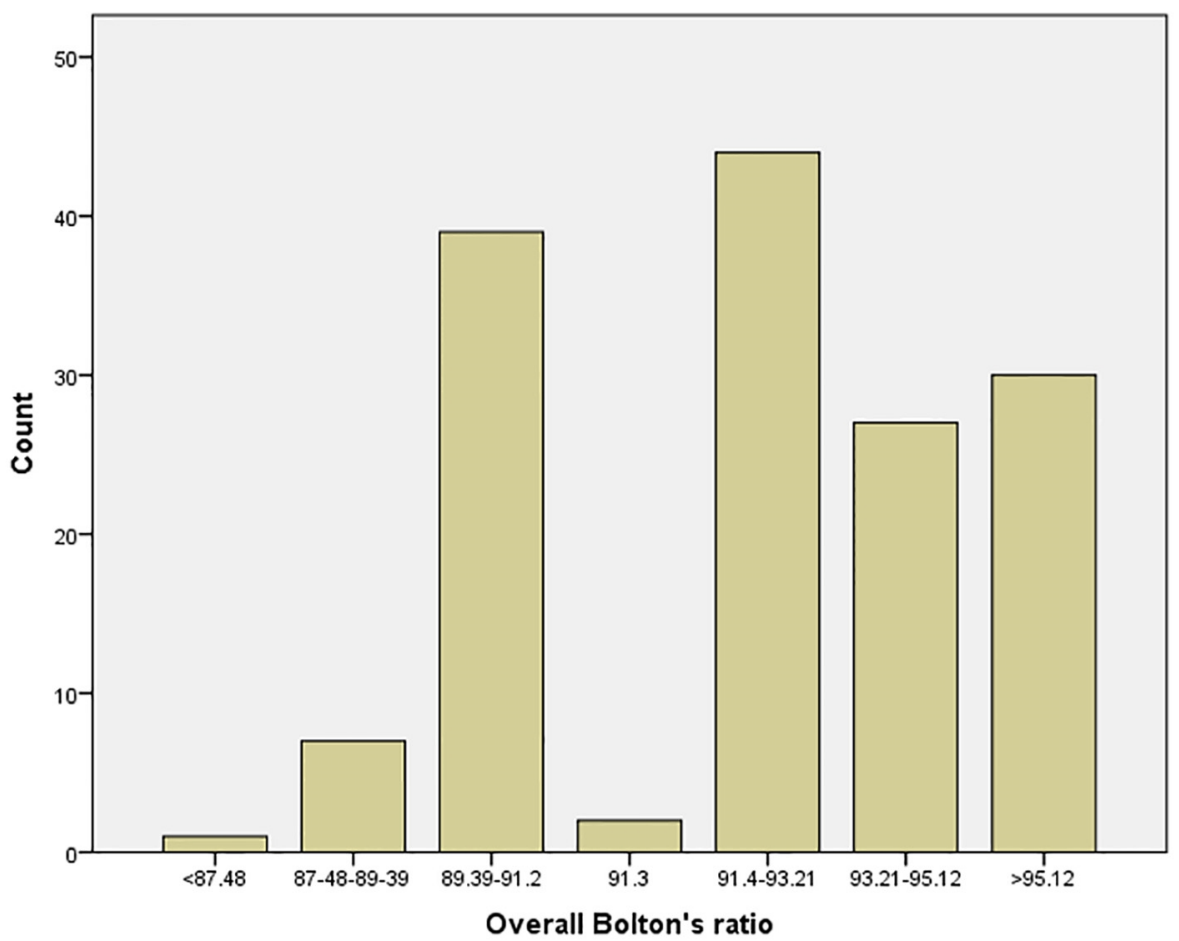

Figure 2. The distribution of overall tooth ratios in this study categorized by Bolton's original mean and SD

\subsection{Reliability}

Inter-examiner analysis revealed that there was no significant difference between the two sets of measurements related to "anterior-ratio", but some slight differences could be found in relation to "overall-ratio" (Table 2). The Pearson correlation coefficients are extremely high enough to accept the fact that these differences are not seriously significant to have influence on the results. Intra-examiner evaluation reveals no significant differences for both examiners (Table 3). The correlation coefficients were extremely high for both examiners.

Table 2. Inter examiner error analysis

\begin{tabular}{|l|l|l|l|l|}
\hline & Sum of Ranks & p-value & Correlation & p-value of correlation \\
\hline Inter examiner/ ant ratio & 35.50 & 0.415 & 0.939 & $<0.001$ \\
\hline Inter examiner/TBI & 49.00 & 0.028 & 0.928 & $<0.001$ \\
\hline
\end{tabular}

Table 3. Intra examiner error analysis

\begin{tabular}{|l|l|l|l|l|}
\hline & Sum of Ranks & p-value of Wilcoxon & Correlation & p-value of Correlation \\
\hline Intra examiner1/ ant ratio & 22.00 & 0.575 & 0.915 & $<0.001$ \\
\hline Intra examiner1/TBI & 29.00 & 0.878 & 0.874 & $<0.001$ \\
\hline Intra examiner2/ ant ratio & 22.00 & 0.581 & 0.923 & $<0.001$ \\
\hline Intra examiner2/TBI & 28.00 & 0.885 & 0.884 & $<0.001$ \\
\hline
\end{tabular}

\subsection{Quick Look Test}

The result of comparison between the quick look test and TSDs beyond 2SDs of Bolton's mean for ABI is shown in Table 4 . The specificity and sensitivity of the quick look test were $34.1 \%$ and $96 \%$ by order, as demonstrated in Figures 3 and 4.

Table 4. Summary of Sensitivity and Specificity for anterior TSD and the Quick Look test

\begin{tabular}{|l|l|l|}
\hline Variable & Anterior TSD & Quick look test \\
\hline Sample size & 69 & 69 \\
\hline Area under the ROC curve & 0.663 & 0.663 \\
\hline Standard error & 0.070 & 0.066 \\
\hline Cut off point & $>76.85$ & $\leq 0.12$ \\
\hline Sensitivity (\%) & 96.0 & 96.0 \\
\hline Specificity (\%) & 34.1 & 34.1 \\
\hline
\end{tabular}




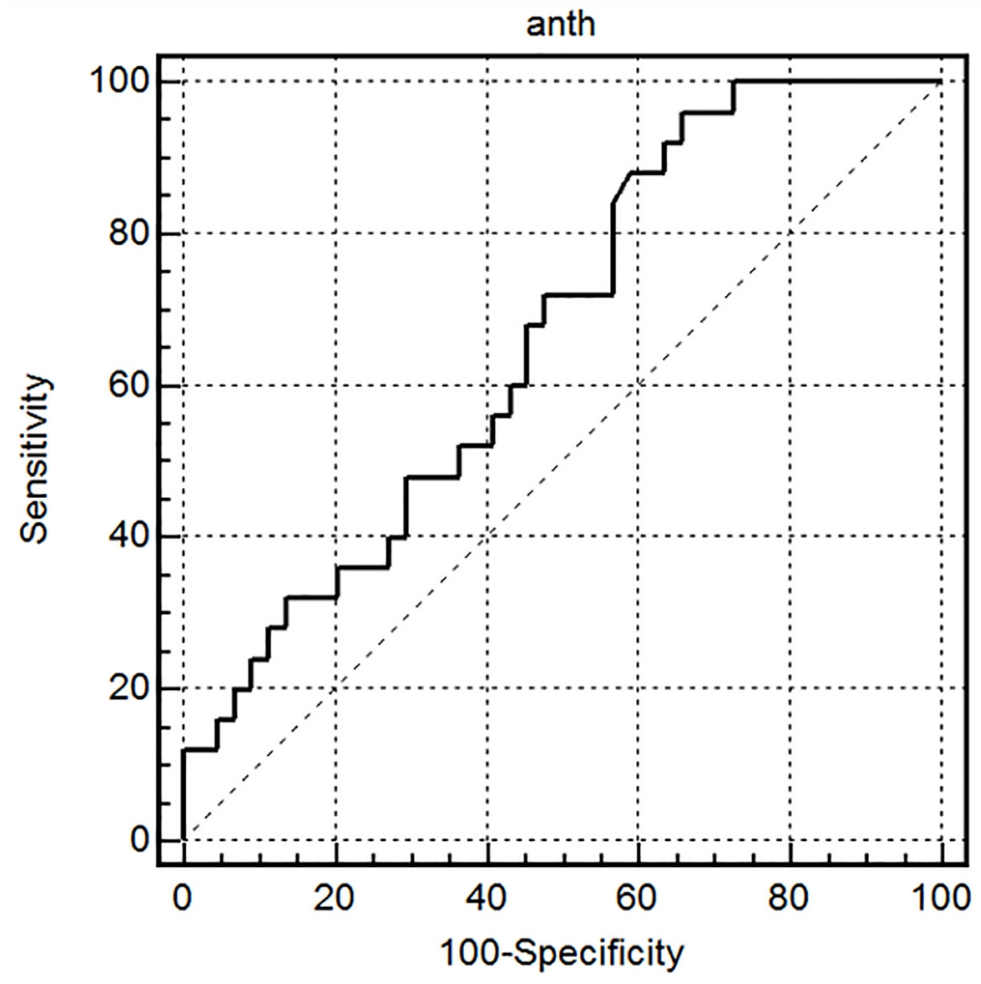

Figure 3. ROC curve analysis of anterior TSD

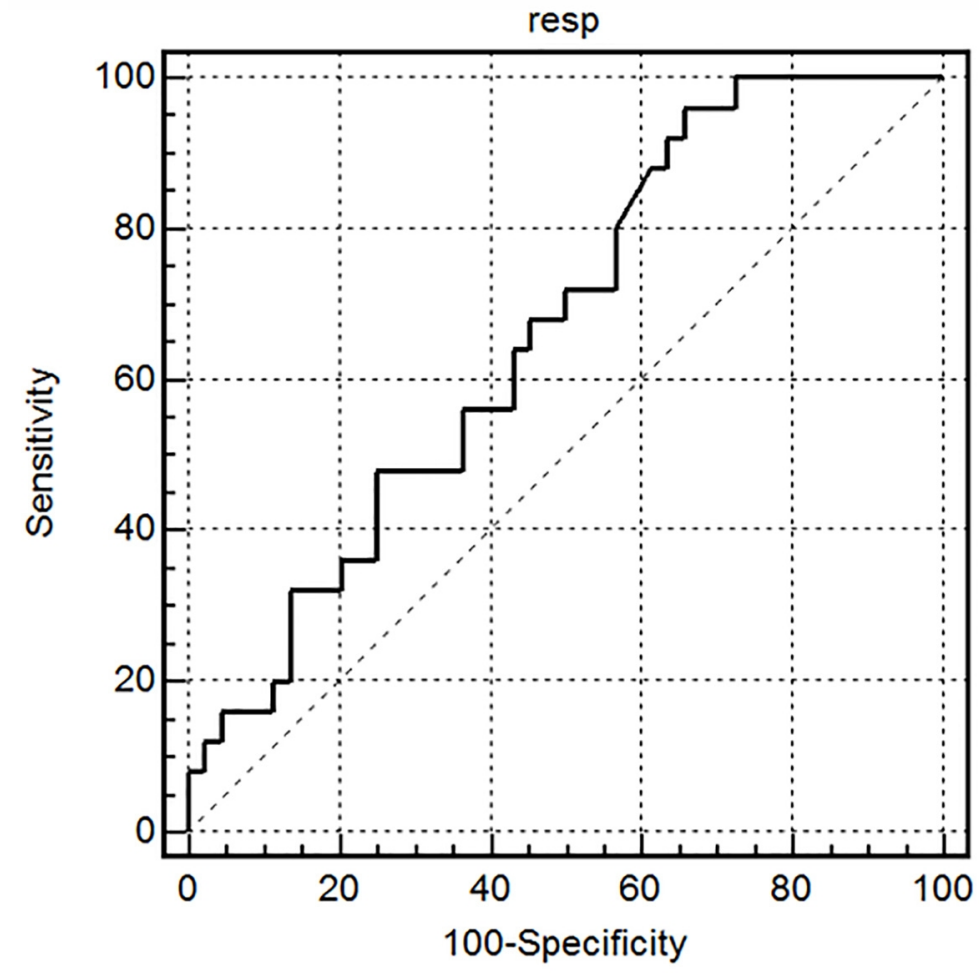

Figure 4. ROC curve analysis of the Quick Look test

\section{Discussion}

Anterior and total Bolton index values are not similar in different populations (3, 7-10). Some Iranian studies presented the mean values of Bolton indices for local populations (11-13). This study tried to provide the prevalence 
of clinically significant TSD beyond 2SDs of Bolton's means for an Iranian population and also to establish a scientific approval of the reliability of the visual judgment of TSD, if possible. For this purpose, we designated an expert orthodontist to check the presence of anterior tooth size discrepancy following Proffit's suggestion (6) and then the sensitivity and specificity of the measurements were evaluated to see how concise this visual estimation would be. Some studies evaluating tooth size discrepancies for different societies are demonstrated in Table 5 .

Table 5. Summary of TSD calculated in different populations

\begin{tabular}{|c|c|c|}
\hline Author & Anterior TSD $\%$ & Overall TSD $\%$ \\
\hline Crosby and Alexander ${ }^{(10)}$ & 22.9 & - \\
\hline Freeman et al..$^{(16)}$ & 30.6 & 13.5 \\
\hline Santoro et al..$^{(9)}$ & 28.0 & 11.0 \\
\hline Araujo and Souki ${ }^{(2)}$ & 22.7 & - \\
\hline Bernabe $^{\swarrow}$ et al. ${ }^{(17)}$ & 20.5 & 5.4 \\
\hline Siti Othman et al. ${ }^{(22)}$ & 17.4 & 5.4 \\
\hline Sharma et al. ${ }^{(18)}$ & 24.0 & 8.0 \\
\hline Uysal T et al. ${ }^{(3)}$ & 21.3 & 18.0 \\
\hline Present study & 34.7 & 20.7 \\
\hline
\end{tabular}

The overall TSD from Bolton's mean \pm 2 SDs reported in the present study, (20.7\%), was much higher than previous studies which declared the TBI in the range of 5.4 to $18 \%(3,14-18)$. The present study also revealed that $34.7 \%$ of the sample had an ABI with a clinically significant deviation from Bolton's mean \pm 2 SDs, which is almost inconsistent with the same values of other populations (Table 5). Uysal and Sari found $21.3 \%$ of ABI and $18 \%$ of TBI to be outside 2SDs from Bolton's mean (3). Ter-Poghosyan showed that about $28.75 \%$ and $11.25 \%$ of an Armenian population had anterior and overall TSD respectively (19). A study conducted by Crosby and Alexander found that $22.9 \%$ of the subjects showed an ABI with a clinically significant deviation from Bolton's mean that was greater than 2 standard deviations (10). Breaking the 34.7\% anterior TSD into $32 \%$ mandibular excess plus $2.7 \%$ excess of maxilla, displayed that that most of the clinically significant TSDs were related to mandibular excess in comparison to maxillary excess, which is not accordant with some other studies; this amount of prevalence of mandibular excess in ABI in comparison to maxilla is much higher than what was reported by Freeman et al. (16), Santoro (9) and Araujo and Souki (2). The latter reported 19.7\% of mandibular anterior excess compared to $10.8 \%$ of maxillary excess. However, Sharma et al. reporting $24 \%$ of anterior TSD greater than 2SDs from Bolton's mean, similarly displayed that there were twice as many cases of mandibular tooth excess than maxillary ones in a population from North India (18). These findings could be ethnic-specific. Regragui et al. have also concluded that in a large number of anterior discrepancies, it was "maxilla" that led to TSD. They attributed most of the anterior abnormalities to the small size of maxillary lateral incisors (14). The original Bolton's study was on 55 cases with ideal occlusion and it was only pertinent to white females (1). On the other hand, Narender Hasija et al. recently mentioned that Bolton's ratios are utilizable for all cases irrespective of types of malocclusion, and essential for investigation of post-treatment stability (20). Another study in a Moroccan population has approved this conclusion by achieving the same results (21). Using the standard deviation of Bolton's mean to determine the prevalence of TSDs could lead to underestimation (15). One way for orthodontists to get a better estimation of the clinical significance of TSDs is to focus more on the actual size of the discrepancies than ratios. In this regard, Proffit suggested that TSDs more than $1.5 \mathrm{~mm}$ could be considered clinically significant and need therapeutic interventions (6) Some studies chose $1.5 \mathrm{~mm}$ as a clinically significant TSD $(7,17)$, this study revealed that almost half of the patients (45.4\%) had clinically significant TSDs, if considering $1.5 \mathrm{~mm}$ as the index. Othman et al. also used $1.5 \mathrm{~mm}$ as the level of significance for TSD and according to this criterion, $42 \%$ of patients had total maxillary excess and $36 \%$ of lower arch had excess (22). These values are much higher than the results of our study. On the other hand, Othman and Harradine stated that $1.5 \mathrm{~mm}$ discrepancy is only $0.75 \mathrm{~mm}$ per side and could lead to hesitation for any adding or reducing of tooth mass (15). Moreover the labiolingual thickness of teeth can also influence the fitness of occlusion as stated by Bolton (1). As recommended by other studies, a threshold of $2 \mathrm{~mm}$ seems a more acceptable index for requiring interventions $(17,18,22)$. Using quick look to estimate TSD in the anterior segments, we obtained a very reliable cut off point of minus $0.12 \mathrm{~mm}$ as a predictive index in screening stage (Table 4). The sensitivity of the quick look method was $96 \%$ and the specificity of it was $34.1 \%$ (Table 4). Although this specificity seems low, the high sensitivity could bring acceptable predictions. This means even if at this screening point, we consider a patient with good intermaxillary tooth size relationships as a patient owning significant tooth size discrepancies by mistake, no invasive intervention would be detected in accurate Bolton analysis on the casts, and 
the occlusion would be perfect spontaneously at the finishing stage with no need for further interventions. On the other hand, a high sensitivity would put an emphasis on the need for an accurate investigation of anterior TSD related to maxillary tooth mass deficiencies in relation to mandible. However, in the only study which compared the visual judgment with TSD measurements, the amount of sensitivity and specificity was lower in comparison to this study (22). This may be caused by the fact that they compared the total TSD to the "Eyeball Estimation" visual judgment; as the result of our study also revealed, the inter examiner error was higher for overall TSD measurements in comparison to anterior TSD measurements. Moreover, the precision and proficiency of the investigator may impact the result. A limitation of the study was to define the measures manually which would reduce the accuracy of the measurements. To overcome this problem, we would suggest the newly-made formulae using 3D scanning technology, the new Johnson/Bailey analysis developed by a team at the University of California, San Francisco (UCSF) which provides more specific ratios utilizing more clinically relevant functional arch components derived from dental cusp-fossa interdigitation (23).

\section{Conclusions}

Due to higher prevalence of TSD \pm 2 SDs of Bolton's means in the Iranian population compared with other studies, this study advises Iranian orthodontists to be more accurate and sharp-eyed on this issue in the initial diagnosis. This article also states that the visual estimation based on the size of lateral incisors will not let you down in recognition of anterior tooth size discrepancy at the screening stage. For future studies, we recommend determining the prevalence of clinically significant tooth size discrepancy in different sagittal and vertical skeletal malocclusions.

\section{Acknowledgments:}

The authors thank the vice-chancellery of Shiraz University of Medical Sciences, for supporting the research (Grant\# 6143). Also, the authors thank Dr. Mehrdad Vossoughi from the Dental Research Development Center, for the statistical analysis. The authors also gratefully acknowledge the Electronic Physician journal for its great technical comments to improve the quality of the manuscript.

\section{Conflict of Interest:}

There is no conflict of interest to be declared.

\section{Authors' contributions:}

All authors contributed to this project and article equally. All authors read and approved the final manuscript.

\section{References:}

1) Bolton WA. Disharmony In Tooth Size And Its Relation To The Analysis And Treatment Of Malocclusion. Angle Orthod. 1958; 28(3): 113-30.

2) Araujo E, Souki M. Bolton anterior tooth size discrepancies among different malocclusion groups. Angle Orthod. 2003; 73(3): 307-13.

3) Uysal T, Sari Z. Intermaxillary tooth size discrepancy and mesiodistal crown dimensions for a Turkish population. Am J Orthod Dentofacial Orthop. 2005; 128(2): 226-30. doi: 10.1016/j.ajodo.2004.04.029.

4) Bolton WA. The clinical application of a tooth-size analysis. Am J Orthod. 1962; 48(7): 504-29. doi: 1016/0002-9416(62)90129-X.

5) Fields HW. Orthodontic-restorative treatment for relative mandibular anterior excess tooth-size problems. Am J Orthod. 1981; 79(2): 176-83. doi: 10.1016/0002-9416(81)90315-8.

6) Proffit W, Fields H. Contemporary orthodontics. 3rd edn. St Louis: Mosby; 2000.

7) Lew K, Keng S. Anterior crown dimensions and relationship in an ethnic Chinese population with normal occlusions. Aust Orthod J. 1991; 12(2): 105. PMID: 1843792.

8) Ta TA, Ling JY, Hägg U. Tooth-size discrepancies among different occlusion groups of southern Chinese children. Am J Orthod Dentofacial Orthop. 2001; 120(5): 556-8. doi: 10.1067/mod.2001.118998. PMID: 11709674.

9) Santoro M, Ayoub ME, Arthur Pardi V, Cangialosi TJ. Mesiodistal crown dimensions and tooth size discrepancy of the permanent dentition of Dominican Americans. Angle Orthod. 2000; 70(4): 303-7. PMID: 10961780.

10) Crosby DR, Alexander CG. The occurrence of tooth size discrepancies among different malocclusion groups. Am J Orthod Dentofacial Orthop. 1989; 95(6): 457-61. doi: 10.1016/0889-5406(89)90408-3.

11) Kachoei M, Ahangar-Atashi MH, Pourkhamneh S. Bolton's intermaxillary tooth size ratios among Iranian schoolchildren. Med Oral Patol Oral Cir Bucal. 2011; 16(4): e568-72. 
12) Mirzakouchaki B, Shahrbaf S, Talebiyan R. Determining tooth size ratio in an Iranian-Azari population. J Contemp Dent Pract. 2007; 8(7): 86-93.

13) Fattahi HR, Pakshir HR, Hedayati Z. Comparison of tooth size discrepancies among different malocclusion groups. Eur J Orthod. 2006; 28(5): 491-5. doi: 10.1093/ejo/cj1012.

14) Regragui S, Azaroual F, Tlemsani H, Aalloula Eh. Étude des dimensions des incisives latérales: responsabilité dans les dysharmonies dento-dentaires antérieures. Int Orthod. 2009; 7(3): 277-85. doi: 10.1016/S1761-7227(09)73503-X.

15) Othman S, Harradine N. Tooth-size discrepancy and Bolton's ratios: a literature review. J Orthod. 2006; 33(1): 45-51. doi: 10.1179/146531205225021384.

16) Freeman JE, Maskeroni A, Lorton L. Frequency of Bolton tooth-size discrepancies among orthodontic patients. Am J Orthod Dentofacial Orthop. 1996; 110(1): 24-7. doi: 10.1016/S0889-5406(96)70083-5. PMID: 8686674.

17) Bernabe E, Major PW, Flores-Mir C. Tooth-width ratio discrepancies in a sample of Peruvian adolescents. Am J Orthod Dentofacial Orthop. 2004; 125(3): 361-5. doi: 10.1016/j.ajodo.2003.04.008.

18) Sharma R, Kumar S, Singla A. Prevalence of tooth size discrepancy among North Indian orthodontic patients. Contemp Clin Dent. 2011; 2(3): 170-5. doi: 10.4103/0976-237X.86445. PMCID: PMC3214522.

19) Ter-Poghosyan HY, Jalladyan NV, Verma S. Bolton tooth size discrepancies in different malocclusions. New Armenian Medical Journal. 2016; 10(1): 82-6.

20) Hasija N, Bala M, Goyal V. Estimation of Tooth Size Discrepancies among Different Malocclusion Groups. Int J Clin Pediatr Dent. 2014; 7(2): 82. doi: 10.5005/jp-journals-10005-1242. PMCID: PMC4212162.

21) Zerouaoui MF, Bahije L, Zaoui F, Regragui S. Study of variations of the Bolton index in the Moroccan population depending on angle malocclusion class. Int Orthod. 2014; 12(2): 213-21. doi: 10.1016/j.ortho.2014.03.003.

22) Othman S, Harradine N. Tooth size discrepancies in an orthodontic population. Angle Orthod. 2007; 77(4): 668-74. doi: 10.2319/031406-102.

23) Bailey E, Nelson G, Miller AJ, Andrews L, Johnson E. Predicting tooth-size discrepancy: A new formula utilizing revised landmarks and 3-dimensional laser scanning technology. Am J Orthod Dentofacial Orthop. 2013; 143(4): 574-85. doi: 10.1016/j.ajodo.2012.09.022. 